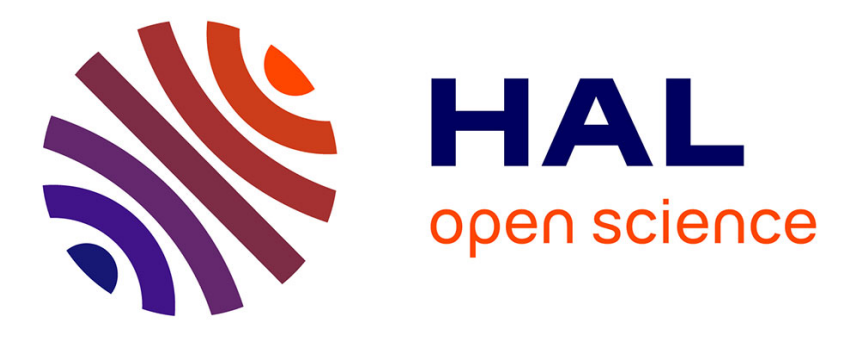

\title{
Temporal filtering: A consistent formalism for seamless hybrid RANS-LES modeling in inhomogeneous turbulence
}

Atabak Fadai-Ghotbi, Christophe Friess, Remi Manceau, Thomas B Gatski, Jacques Borée

\section{To cite this version:}

Atabak Fadai-Ghotbi, Christophe Friess, Remi Manceau, Thomas B Gatski, Jacques Borée. Temporal filtering: A consistent formalism for seamless hybrid RANS-LES modeling in inhomogeneous turbulence. International Journal of Heat and Fluid Flow, 2010, 31 (3), pp.378-389. 10.1016/j.ijheatfluidflow.2009.12.008 . hal-02136406

\section{HAL Id: hal-02136406 \\ https://hal.science/hal-02136406}

Submitted on 24 May 2019

HAL is a multi-disciplinary open access archive for the deposit and dissemination of scientific research documents, whether they are published or not. The documents may come from teaching and research institutions in France or abroad, or from public or private research centers.
L'archive ouverte pluridisciplinaire HAL, est destinée au dépôt et à la diffusion de documents scientifiques de niveau recherche, publiés ou non, émanant des établissements d'enseignement et de recherche français ou étrangers, des laboratoires publics ou privés.

\section{(ㅇ)(1) $\$$}

Distributed under a Creative Commons Attribution - NonCommercial - NoDerivatives 44.0 


\title{
Temporal filtering: a consistent formalism for seamless hybrid RANS-LES modeling in inhomogeneous turbulence
}

\author{
Atabak Fadai-Ghotbi, Christophe Friess, Rémi Manceau ${ }^{\dagger}$, \\ Thomas B. Gatski ${ }^{1}$, Jacques Borée
}

Department of Fluid Flow, Heat Transfer and Combustion

Institute PPRIMME

CNRS-University of Poitiers-ENSMA

SP2MI, Bd Marie et Pierre Curie, BP 30179

86962 Futuroscope Chasseneuil Cedex, France

${ }^{\dagger}$ Corresponding author:

remi.manceau@univ-poitiers.fr

tel: $(+33)$ 549496927; fax: $(+33) 549496968$

\footnotetext{
${ }^{1}$ Also: Center for Coastal Physical Oceanography and Ocean, Earth and Atmospheric Sciences, Old Dominion University, Norfolk, VA 23529
} 


\begin{abstract}
A consistent formalism is developed for seamless hybrid RANSLES models in inhomogeneous, stationary flows, based on Eulerian temporal filtering. The issues of Galilean invariance of the filtering process and consistency with the Reynolds average are addressed. The similarity of the RANS and TLES equations suggests the use of the same form of model for the two limiting approaches. The inconsistency of the existing TLES models with the RANS limit leads to the choice of the opposite strategy: adapting a RANS model to the TLES limit. The method proposed to achieve this adaptation is the Temporal Partially Integrated Transport Model (TPITM), a temporal version of the spatial PITM. The applicability of the method is shown by performing channel flow simulations using transport equations for the subfilter stresses, derived from the Elliptic-Blending Reynolds-Stress RANS Model (EB-RSM). Finally, the fact that the temporal filter width can be implicitly defined by the associated spatial filter width suggests that most of the unsteady approaches used in everyday applications, such as DES, SAS, URANS, among others, can be regarded as temporally filtered approaches.
\end{abstract}

Keywords: Eulerian Temporal filter; TLES; T-PITM; URANS; DES; Ellipticblending Reynolds-stress model. 


\section{Introduction}

Unsteady computations of turbulent flows are of great relevance in an industrial context, and concern, e.g., noise emissions, structure vibrations or thermal fatigue. Since LES (Large-Eddy Simulation) is too CPU-demanding for many complex industrial applications, a multitude of unsteady low-cost strategies have gained prominence over the last decade (see, e.g., Fröhlich and von Terzi, 2008; Sagaut et al., 2006). Some of these models can be described as seamless hybrid RANS-LES models, in the sense that the computation progressively transitions from a RANS (Reynolds-Averaged Navier-Stokes) model in some regions of the flow, particularly in the near-wall zones, to an LES in other regions where explicit computation of the large-scale structures is required. In statistically homogeneous flows, such a model can be seen as an LES with a filter width $\Delta_{S}$ continuously going to infinity or, equivalently, as an LES with a cutoff wavenumber $\kappa_{c}=\pi / \Delta_{S}$ continuously going to zero - a limit that corresponds formally to the RANS approach. However, the majority of flows of practical relevance are inhomogeneous, and in that case such models suffer from an important conceptual weakness due to the inherently different concepts underlying LES and RANS models: the former give spatially filtered fields; whereas, the latter give long-time averaged fields.

In order to develop a consistent seamless hybrid RANS-LES model in inhomogeneous flows, an Eulerian temporal filtering approach (Pruett, 2000; Pruett et al., 2003) within the LES formalism can be used so that the discussion is now within the context of a TLES (Temporal Large-Eddy Simulation) approach. In this paper a consistent seamless hybrid RANS-LES model for incompressible, stationary, inhomogeneous flows is developed by using an Eulerian temporal filtering approach consistent with the TLES formalism. The constraint of stationarity, i.e., the statistical independence with respect to a shift in time, is not too restrictive, since many flows of practical importance are stationary. This class includes all the flows for which the boundary conditions are not varying in time. In particular, although it can be a matter of philosophical debate, since the vortex shedding appearing in some simple turbulent flows, such as 2D wakes, is not periodic in time (e.g., Ma et al., 2000; Perrin et al., 2007), such flows are considered herein as stationary processes, rather than cyclostationary processes (Antoni, 2009). The case of boundary conditions varying with a time-scale much larger than the time-scale of turbulence can also be considered as eligible to the application of the present methodology, by replacing the constraint of an infinite filter width with the constraint of a filter width much larger than the turbulent time scale.

In section 2, the time-filtering process is presented that satisfies the property of Galilean invariance and the consistency with the Reynolds average. The 
following sections are devoted to the modeling of the subfilter stress in the temporally filtered momentum equation. Section 3 analyzes the behavior of TLES models making explicit use of the filter (Pruett, 2000; Pruett et al., 2003; Tejada-Martinez et al., 2007) in the limit of infinitely large temporal filters. The inherent limitations of these models lead to the proposal, in section 4, of a new approach, the so-called Temporal Partially-Integrated Transport Model (TPITM), which is a transposition to temporal filtering of the PITM approach (Schiestel and Dejoan, 2005; Chaouat and Schiestel, 2005), based on an analysis in the frequency domain, and enables the adaptation of any RANS model to the context of hybrid RANS/TLES. Section 5 then presents the adaptation of a particular model, the Elliptic Blending Reynolds-Stress model (EB-RSM), which leads to a hybrid RANS/TLES based on transport equations for the subfilter stresses, and its application to the case of a channel flow at $R e_{\tau}=395$. Finally, section 6 discusses the possibility of providing a consistent interpretation of the usual eddy-resolving methods, such as DES, SAS, OES, URANS, among others, within the framework of temporal filtering.

\section{Toward a consistent formalism}

In this section, a Galilean invariant, time filtering operation is defined that can be applied in the partitioning of the flow variables into resolved and unresolved (subfilter) parts. The consistency of the filter with the Reynolds average in the limit of an infinite filter width and the formal similarity between the filtered and Reynolds-averaged equations form the foundation for the development in the following sections of a unified model able to bridge RANS and LES.

\subsection{Definition of the time-filtering process}

In order to decompose the instantaneous velocity $\mathbf{u}^{*}$ into a filtered, resolved part $\widetilde{\mathbf{U}}=<\mathbf{u}^{*}>$, and a residual part $\mathbf{u}^{\prime \prime}$, the resolved part is defined by using a filter $<.>$ expressed in the general form

$$
<\mathbf{u}^{*}>(\mathbf{x}, t)=\iint \mathcal{G}\left(\mathbf{x}^{\prime}-\boldsymbol{\xi}\left(\mathbf{x}, t^{\prime}-t\right), t^{\prime}-t\right) \mathbf{u}^{*}\left(\mathbf{x}^{\prime}, t^{\prime}\right) \mathrm{d} \mathbf{x}^{\prime} \mathrm{d} t^{\prime}
$$

with a kernel of the form

$$
\mathcal{G}\left(\mathbf{x}^{\prime}-\boldsymbol{\xi}\left(\mathbf{x}, t^{\prime}-t\right), t^{\prime}-t\right)=\delta\left(\mathbf{x}^{\prime}-\boldsymbol{\xi}\left(\mathbf{x}, t^{\prime}-t\right)\right) G_{\Delta_{T}}\left(t^{\prime}-t\right)
$$


with $\Delta_{T}$ the temporal filter width. For instance, the exponential filter used by Pruett et al. (2003) and Tejada-Martinez et al. (2007) reads

$$
\mathcal{G}\left(\mathbf{x}^{\prime}-\mathbf{x}, t^{\prime}-t\right)=\delta\left(\mathbf{x}^{\prime}-\mathbf{x}\right) \frac{1}{\Delta_{T}} \exp \left(\frac{t^{\prime}-t}{\Delta_{T}}\right) H\left(t-t^{\prime}\right)
$$

where $H$ is the Heaviside function. In this case, the spatial part of the filter is the familiar Dirac function, i.e., $\boldsymbol{\xi}\left(\mathbf{x}, t^{\prime}-t\right)=\mathbf{x}$.

The introduction of $\boldsymbol{\xi}\left(\mathbf{x}, t^{\prime}-t\right)$, with $\boldsymbol{\xi}(\mathbf{x}, 0)=\mathbf{x}$, in the definition of the filter aims at ensuring that the filtering operation preserve the translational, or Galilean, invariance, which is not the case for all the filters (Pruett, 2000).

\subsection{Galilean invariance of the filter}

In the development of a seamless hybrid temporally filtered methodology that is capable of spanning the range of solution methodologies from DNS to RANS, it is necessary to insure that the resulting equations retain the same invariance properties as the Navier-Stokes and Reynolds averaged equations. Consider the translational transformation of the spatial frame of reference $\mathbf{x}$ given by

$$
\mathbf{x}^{\bullet}=\mathbf{x}-\mathbf{U}_{0} t
$$

where $\mathbf{x}^{\bullet}$ is the coordinate vector in the translating frame, and $\mathbf{U}_{0}$ is a constant displacement velocity. In order to retain Galilean invariance for the filtered equations, it is necessary that

$$
<\mathbf{u}^{* \bullet}>\left(\mathbf{x}^{\bullet}, t\right)=<\mathbf{u}^{*}>(\mathbf{x}, t)-\mathbf{U}_{0},
$$

where $\mathbf{u}^{* \bullet}$ is the velocity in the translating frame.

For the case where the standard filter $\boldsymbol{\xi}(\mathbf{x}, \tau)=\mathbf{x}$ is applied, which reads $\boldsymbol{\xi}^{\bullet}\left(\mathrm{x}^{\bullet}, \tau\right)=\mathrm{x}^{\bullet}$ in the translating frame, the filtered velocity reads

$$
\begin{aligned}
<\mathbf{u}^{* \bullet}>\left(\mathbf{x}^{\bullet}, t\right) & =\int G_{\Delta_{T}}(\tau) \mathbf{u}^{* \bullet}\left(\mathbf{x}^{\bullet}, t+\tau\right) \mathrm{d} \tau \\
& =\int G_{\Delta_{T}}(\tau) \mathbf{u}^{*}\left(\mathbf{x}^{\bullet}+(t+\tau) \mathbf{U}_{0}, t+\tau\right) \mathrm{d} \tau-\mathbf{U}_{0} \\
& =\int G_{\Delta_{T}}(\tau) \mathbf{u}^{*}\left(\mathbf{x}+\mathbf{U}_{0} \tau, t+\tau\right) \mathrm{d} \tau-\mathbf{U}_{0} \\
& \neq \int G_{\Delta_{T}}(\tau) \mathbf{u}^{*}(\mathbf{x}, t+\tau) \mathrm{d} \tau-\mathbf{U}_{0} \\
& \neq<\mathbf{u}^{*}>(\mathbf{x}, t)-\mathbf{U}_{0},
\end{aligned}
$$


such that the transformation is not Galilean invariant. This is the relation obtained by Pruett (2000) in assessing a Doppler effect on time filtered variables. This lack of translational invariance can be remedied by considering a new, generalized definition for $\boldsymbol{\xi}$ parametrized by a reference velocity

$$
\boldsymbol{\xi}(\mathbf{x}, \tau)=\mathbf{x}+\mathbf{V}_{\text {ref }} \tau,
$$

which transforms as $\boldsymbol{\xi}^{\bullet}\left(\mathbf{x}^{\bullet}, \tau\right)=\mathbf{x}^{\bullet}+\mathbf{V}_{\text {ref }}^{\bullet} \tau$ in the translating frame. The filtered velocity then becomes

$$
\begin{aligned}
<\mathbf{u}^{* \bullet}>\left(\mathbf{x}^{\bullet}, t\right)= & \int G_{\Delta_{T}}(\tau) \times \\
& \mathbf{u}^{*}\left(\mathbf{x}^{\bullet}+\mathbf{V}_{\mathrm{ref}}^{\bullet} \tau+(t+\tau) \mathbf{U}_{0}, t+\tau\right) \mathrm{d} \tau-\mathbf{U}_{0} \\
& =\int G_{\Delta_{T}}(\tau) \mathbf{u}^{*}\left(\mathbf{x}^{\bullet}+\mathbf{U}_{0} t+\tau \mathbf{V}_{\text {ref }}, t+\tau\right) \mathrm{d} \tau-\mathbf{U}_{0} \\
& =\int G_{\Delta_{T}}(\tau) \mathbf{u}^{*}(\boldsymbol{\xi}, t+\tau) \mathrm{d} \tau-\mathbf{U}_{0} \\
& =<\mathbf{u}^{*}>(\mathbf{x}, t)-\mathbf{U}_{0},
\end{aligned}
$$

such that now the desired Galilean invariant transformation property holds. These results show that, in order to preserve the Galilean invariance, the filter $\mathcal{G}$ must be defined using $\boldsymbol{\xi}(\mathbf{x}, \tau)=\mathbf{x}+\mathbf{V}_{\text {ref }} \tau$, i.e., the temporal kernel $G_{\Delta_{T}}$ of the filter is necessarily applied at a location moving with some reference velocity. In order to unambiguously define the filter, this reference velocity $\mathbf{V}_{\text {ref }}$ must be related to the flow configuration, e.g., the boundary conditions (for instance, the velocity of an obstacle).

Moreover, in the limit of an infinite filter width $\Delta_{T}$, this definition of the filter ensures, in all the inertial frames, the compatibility with the Reynolds average that is Galilean invariant. On the contrary, the standard definition goes to the long-time average when $\Delta_{T} \rightarrow \infty$, which does not satisfy Galilean invariance, as noted by Speziale (1987). For instance, with a kernel of top-hat type and $\boldsymbol{\xi}$ defined by Eq. (7), the generalized filtering operator (1) becomes

$$
\mathcal{G}\left(\mathbf{x}^{\prime}-\boldsymbol{\xi}\left(\mathbf{x}, t^{\prime}-t\right), t^{\prime}-t\right)=\delta\left(\mathbf{x}^{\prime}-\mathbf{x}-\mathbf{V}_{\text {ref }}\left(t^{\prime}-t\right)\right) \frac{1}{\Delta_{T}} H\left(t^{\prime}-t+\Delta_{T}\right) H\left(t-t^{\prime}\right),
$$

and the generalized long-time averaging defined by

$$
\left[\mathbf{u}^{*}\right]_{\left\{\infty ; \mathbf{V}_{\text {ref }}\right\}}(\mathbf{x}, t)=\lim _{\Delta_{T} \rightarrow \infty}<\mathbf{u}^{*}>(\mathbf{x}, t)
$$

inherits the Galilean invariance from the filter. The definitions of the filter (1) and the associated generalized long-time average (10) thus provide the 
consistent formalism for seamless hybrid RANS/LES methods (more accurately, hybrid RANS/TLES), for a particular class of flows: the flows that are stationary in a particular reference frame. Therefore, in describing the model development in the remainder of this paper, in order to simplify the analysis, the flow is assumed to belong to this class, the reference frame is the particular frame in which the flow is stationary, and the parameter $\mathbf{V}_{\text {ref }}$ of the filter is zero in this frame.

\subsection{Filtered equations}

The filtered velocity is denoted by $\widetilde{\mathbf{U}}=\left\langle\mathbf{u}^{*}\right\rangle$ and the residual velocity is defined by

$$
\mathbf{u}^{\prime \prime}=\mathbf{u}^{*}-\widetilde{\mathbf{U}} .
$$

Reynolds averaging, or long-time averaging, is denoted by an overbar (. ), and the Reynolds-averaged velocity by $\mathbf{U}=\overline{\mathbf{u}^{*}}$. The fluctuating part of the filtered velocity is defined by

$$
\mathbf{u}^{\prime}=\widetilde{\mathbf{U}}-\mathbf{U}
$$

and the total fluctuation by

$$
\mathbf{u}=\mathbf{u}^{*}-\mathbf{U}=\mathbf{u}^{\prime}+\mathbf{u}^{\prime \prime}
$$

The same notation is used for the decomposition of the instantaneous pressure

$$
p^{*}=\widetilde{P}+p^{\prime \prime}=P+p=P+p^{\prime}+p^{\prime \prime} .
$$

Assuming, as in spatial LES, that the filter commutes with the differential operators, which is exact if the temporal filter width $\Delta_{T}$ is constant, the incompressibility constraint for the instantaneous field is inherited by the filtered field

$$
\frac{\partial \widetilde{U}_{k}}{\partial x_{k}}=0
$$

and the filtered momentum equation reads

$$
\frac{\partial \widetilde{U}_{i}}{\partial t}+\widetilde{U}_{k} \frac{\partial \widetilde{U}_{i}}{\partial x_{k}}=-\frac{1}{\rho} \frac{\partial \widetilde{P}}{\partial x_{i}}+\nu \frac{\partial^{2} \widetilde{U}_{i}}{\partial x_{j} \partial x_{j}}-\frac{\partial \tau_{i j_{S F S}}}{\partial x_{j}} .
$$

$\tau_{i j_{S F S}}$ is the subfilter-scale (SFS) tensor, defined as the generalized central second moment $\tau_{i j_{S F S}}=\tau\left(u_{i}^{*}, u_{j}^{*}\right)$, where $\tau(a, b)=<a b>-<a><b>$ for 
any variables $a$ and $b$. Similarly to what was shown by Germano (1992) for spatial filtering, the transport equation for the subfilter stress reads

$$
\begin{aligned}
& \frac{\partial \tau_{i j_{S F S}}}{\partial t}+\widetilde{U}_{k} \frac{\partial \tau_{i j_{S F S}}}{\partial x_{k}}=\underbrace{-\frac{\partial \tau\left(u_{i}^{*}, u_{j}^{*}, u_{k}^{*}\right)}{\partial x_{k}}}_{D_{i j_{S F S}}^{T}}+\underbrace{-2 \nu \tau\left(\frac{\partial u_{i}^{*}}{\partial x_{k}}, \frac{\partial u_{j}^{*}}{\partial x_{k}}\right)}_{D_{i j_{S F S}}^{\nu} \frac{\partial^{2} \tau_{i j_{S F S}}}{\partial x_{k} \partial x_{k}}} \\
& \underbrace{-\frac{1}{\rho} \tau\left(u_{i}^{*}, \frac{\partial p^{*}}{\partial x_{j}}\right)-\frac{1}{\rho} \tau\left(u_{j}^{*}, \frac{\partial p^{*}}{\partial x_{i}}\right)}_{\phi_{i j_{S F S}}} \underbrace{-\tau_{i k S F S} \frac{\partial \widetilde{U}_{j}}{\partial x_{k}}-\tau_{j k_{S F S}} \frac{\partial \widetilde{U}_{i}}{\partial x_{k}}}_{P_{i j_{S F S}}},
\end{aligned}
$$

where

$$
\begin{array}{r}
\tau(a, b, c)=<a b c>-<a>\tau(b, c)-<b>\tau(a, c) \\
-<c>\tau(a, b)-<a><b><c>.
\end{array}
$$

This equation is formally identical to the familiar RANS equation for the Reynolds stress $\overline{u_{i} u_{j}}$, which is the basis for the adaptation of a RANS second moment closure to the hybrid RANS/TLES context presented in section 5. $D_{i j_{S F S}}^{T}, D_{i j_{S F S}}^{\nu}, \varepsilon_{i j_{S F S}}, \phi_{i j_{S F S}}$ and $P_{i j_{S F S}}$ represent subfilter-scale turbulent diffusion, viscous diffusion, dissipation, velocity-pressure gradient correlation and production, respectively. The formal similarity of these terms with their RANS counterpart becomes obvious with an idempotent filter: for instance, in this case, the SFS turbulent diffusion term reads

$$
D_{i j_{S F S}}^{T}=-\frac{\partial<u_{i}^{\prime \prime} u_{j}^{\prime \prime} u_{k}^{\prime \prime}>}{\partial x_{k}}
$$

One of the advantages of Eulerian temporal filtering in the context of stationary flows is that the following property is satisfied

$$
\overline{<\mathbf{u}^{*}>}=\overline{\mathbf{u}^{*}},
$$

such that, as shown by Germano (1992), the total Reynolds stress $\overline{u_{i} u_{j}}$ is exactly decomposed as

$$
\overline{u_{i} u_{j}}=\left(\overline{\tilde{U}_{i} \tilde{U}_{j}}-U_{i} U_{j}\right)+\overline{\tau_{i j_{S F S}}}
$$

The total fluctuating kinetic energy is decomposed as

$$
k=\frac{1}{2} \overline{u_{i} u_{i}}=k_{m}+k_{r}
$$


where the subfilter scale contribution, or modeled part, is

$$
k_{m}=\overline{k_{S F S}}=\frac{1}{2} \overline{\tau_{i i S F S}}
$$

and the resolved part

$$
k_{r}=\frac{1}{2}\left(\overline{\tilde{U}_{i} \tilde{U}_{i}}-U_{i} U_{i}\right)=\frac{1}{2} \overline{u_{i}^{\prime} u_{i}^{\prime}} .
$$

In Eq. (20), the last term in the RHS, $\overline{\tau_{i j_{S F S}}}$, is generally not accounted for in standard LES, since the cutoff wavenumber is located well inside the inertial region. In the case of hybrid methods, the contribution of the subfilter scales can be dominant, and even represent $100 \%$ of the energy in RANS regions. Consequently, an accurate evaluation of this part is necessary, in general through transport equations for the subgrid energy $k_{S F S}$ or, as in Chaouat and Schiestel (2005, 2009); Jakirlić et al. (2009), by directly solving transport equations for the subfilter stress.

The formalism introduced in this section provides an appropriate framework for hybrid methodologies. The definition of the filtering operator, based on a temporal kernel, ensures that the variables $\widetilde{U}_{i}, \widetilde{P}$ and $\tau_{i j_{S F S}}$ consistently and continuously tend to their corresponding RANS counterparts, $U_{i}, P$ and $\overline{u_{i} u_{j}}$, when the temporal filter width goes to infinity. The main issue in this framework is the modeling of the subfilter stress, which must be compatible with all the possible locations of the characteristic cutoff frequency $\omega_{c}$ in the turbulent spectrum: well inside the inertial range (standard TLES), at the RANS limit $\left(\omega_{c}=0\right)$, or, by continuity, in the energetic range, where equilibrium cannot be assumed and complex production and redistribution phenomena play a major role.

The formal similarity of the TLES transport equation for the subfilter stress and the standard RANS equation for the Reynolds stress, moreover suggests that the form of the models used in TLES and RANS regions can be identical, and that a sensitization of the model to the temporal filter width can provide the appropriate adaptation of the level of subfilter stresses in the momentum equation to account for the transition from a TLES to a RANS behavior.

\section{State-of-the-art subfilter stress models}

The first possibility to be considered in order to develop an hybrid RANS/TLES model is to extend an existing TLES subfilter stress model such that it becomes compatible with the RANS limit. For this purpose, the behavior of the models in the limit $\Delta_{T} \rightarrow \infty$ must be investigated. 
Models for the subfilter stresses for LES based on Eulerian temporal filtering have been proposed by Pruett (2000); Pruett et al. (2003); Tejada-Martinez et al. (2007). Since in Smagorinsky-type models, a length scale characterizing the filter is explicitly needed to determine the subfilter viscosity, such models cannot be directly adapted to temporal filtering. Therefore, the authors have used models in which the filter is explicitly used, such as a scale-similarity model (Pruett, 2000; Pruett et al., 2003) or a deconvolution model (Pruett et al., 2003; Tejada-Martinez et al., 2007).

In the former model, the so-called Temporal Scale-Similarity Model (TSSM), the subfilter stress is approximated by

$$
\left.\tau_{i j_{S F S}} \approx<<u_{i}^{*}><u_{j}^{*}>>-<<u_{i}^{*}\right\rangle><<u_{j}^{*}>>,
$$

which is formally equivalent to the model of Bardina et al. (1980) who used a spatial filter. Beside the fact that the scale-similarity hypothesis is valid only in case of equilibrium of the turbulent structures above and below the cutoff frequency, which is only valid in the inertial range of the spectrum, the TSSM model vanishes in the limit $\Delta_{T} \rightarrow \infty$, such that the model is not compatible with the RANS limit. The reason for this behavior is simply that the filtered variables tend to the Reynolds-averaged variables, for which $\left.<<u_{i}^{*}><u_{j}^{*}>>=<<u_{i}^{*}\right\rangle><<u_{j}^{*}>>$ (Kampé De Fériet and Betchov, 1951). Therefore, the best candidate for an extension to the hybrid methodology is the Temporal Approximate Deconvolution Model (TADM) proposed by Pruett et al. (2003), as an adaptation to time filtering of the ADM model (Stolz et al., 2001). This model is based on a spectral partitioning in the frequency domain

$$
\omega \in[0, \infty]=\underbrace{\left[0, \omega_{\Delta_{T}}\right]}_{\begin{array}{c}
\text { Resolved } \\
\text { scales }
\end{array}} \cup \underbrace{\left[\omega_{\Delta_{T}}, \omega_{\Delta t}\right] \cup\left[\omega_{\Delta t}, \infty\right]}_{\begin{array}{c}
\text { Subfilter } \\
\text { scales }
\end{array}}
$$

where $\omega_{\Delta_{T}}$ and $\omega_{\Delta t}$ are the frequencies associated to the temporal width of the filter $\Delta_{T}$ and to the time step $\Delta t$, respectively. Two parts are thus distinguished in the subfilter scales: the resolved subfilter scales (RSFS), in the range $\left[\omega_{\Delta_{T}}, \omega_{\Delta t}\right]$, and the unresolved subfilter scales (USFS) in the range $\left[\omega_{\Delta t}, \infty\right]$.

The RSFS are reconstructed using the temporal approximate deconvolution method

$$
\tau_{i j_{R S F S}} \approx<v_{i} v_{j}>-<v_{i}><v_{j}>
$$

where $v_{i}$ is an approximation of the instantaneous (unfiltered) velocity $u_{i}^{*}$ obtained by applying the approximate inverse filter $G_{\Delta_{T} a}^{-1}$ to the resolved 
velocity

$$
v_{i}=G_{\Delta_{T} a^{*}}^{-1}<u_{i}^{*}>=c_{0}<u_{i}^{*}>+c_{1}<<u_{i}^{*}>>+\cdots+c_{p}<u_{i}^{*}>^{(p+1)}
$$

(* stands for the convolution product). $<.\rangle^{(q)}$ denotes the application of the filter $q$ times, using the primary filter $G_{\Delta_{T}}$.

The remaining contribution to the subfilter stress, the USFS, is obtained by a regularization procedure

$$
\frac{\partial \tau_{i j_{U S F S}}}{\partial x_{j}}=\chi\left(I-G_{\Delta_{T}} * G_{\Delta_{T}}^{-1}\right) *<u_{i}^{*}>,
$$

which is purely dissipative.

The behavior of the TADM model for large values of the temporal filter width $\Delta_{T}$ was investigated by Pruett et al. (2003), who showed that the approximate deconvolution procedure is able to accurately reproduce the exact filtered velocity, provided that the order $q$ of the approximate inverse is sufficiently high. However, they also mentioned that in the limit $\Delta_{T} \rightarrow \infty$, the model is not valid, because the deconvolution procedure assumes that the filter is invertible, which is not the case in this limit: the frequency $\omega_{c}$ characterizing the width of the Fourier transform of the filter $\widehat{G}_{\Delta_{T}}$ goes to zero, such that $\lim _{\Delta_{T} \rightarrow \infty} \widehat{G}_{\Delta_{T}}(\omega)=0$.

Under the criteria that have been established here for the construction of a hybrid RANS/TLES methodology, it is not possible to adapt the TLES formulation just discussed to the hybrid approach since the filter does not tend to the Reynolds average in the limit $\Delta_{T} \rightarrow \infty$. Oftentimes, it is assumed that modification of coefficients is the change necessary in migrating RANS or (T)LES methodologies to a hybrid level. Delineating criteria based on mathematical consistencies, as is done here, can lead to a selection process that preclude what may appear viable hybrid approaches. In the remainder of this article, an alternate strategy is developed: a RANS model is modified by sensitizing the coefficients to the temporal width of the filter.

\section{TPITM methodology}

Within the consistent temporal framework, a new approach is proposed, the Temporal Partially Integrated Transport Model (TPITM), which is an adaptation/extension of the PITM model (Schiestel and Dejoan, 2005; Chaouat and Schiestel, 2005) to the temporal filtering context. The TPITM model is based on a spectral analysis in the frequency domain, in order to guarantee compatibility between the two methodologies - RANS and TLES. 
In section 4.1, the energy partition among filtered and residual scales Eq. (21) is written in the frequency domain. The transport equation for the Eulerian temporal energy spectrum is derived, in order to obtain the transport equation for the subfilter energy $k_{m}$. This equation is the basis for the sensitization, introduced in section 4.2, of the standard RANS dissipation rate equation to the temporal filter width.

\subsection{Energy partition in frequency space}

The turbulent kinetic energy can be written as

$$
k(\mathbf{x})=\frac{1}{2} Q_{i, i}(\mathbf{x}, 0)=\int_{-\infty}^{\infty} \frac{1}{2} \widehat{Q}_{i, i}(\mathbf{x}, \omega) \mathrm{d} \omega=\int_{0}^{\infty} E_{T}(\mathbf{x}, \omega) \mathrm{d} \omega,
$$

where $\widehat{\phi}$ denotes the temporal Fourier transform of $\phi$, defined by

$$
\widehat{\phi}(\mathbf{x}, \omega)=\frac{1}{2 \pi} \int_{-\infty}^{\infty} e^{-i \omega \tau} \phi(\mathbf{x}, \tau) \mathrm{d} \tau .
$$

$Q_{i, j}(\mathbf{x}, \tau)$ is the two-time correlation tensor

$$
Q_{i, j}(\mathbf{x}, \tau)=\overline{u_{i}(\mathbf{x}, t) u_{j}(\mathbf{x}, t+\tau)}
$$

and $E_{T}(\mathbf{x}, \omega)$ the Eulerian temporal turbulent kinetic energy spectrum, given by

$$
E_{T}(\mathbf{x}, \omega)=\widehat{Q}_{i, i}(\mathbf{x}, \omega) .
$$

Introducing $\widehat{G}_{\Delta_{T}}(\omega)$, the Fourier transform of the temporal filter kernel $G_{\Delta_{T}}(\tau)$, the resolved part of the turbulent energy reads

$$
k_{r}(\mathbf{x})=\int_{0}^{\infty} \widehat{G}_{\Delta_{T}}(\omega) \widehat{G}_{\Delta_{T}}^{*}(\omega) E_{T}(\mathbf{x}, \omega) \mathrm{d} \omega
$$

where a star denotes the conjugate, and the residual energy, $k_{m}=k-k_{r}$,

$$
k_{m}(\mathbf{x})=\int_{0}^{\infty}\left[1-\widehat{G}_{\Delta_{T}}(\omega) \widehat{G}_{\Delta_{T}}^{*}(\omega)\right] E_{T}(\mathbf{x}, \omega) \mathrm{d} \omega .
$$

In order to derive the model equation for the dissipation rate, as presented in the next section, the transport equation for $k_{m}$ is required. According to Eq. (33), this equation can be derived from the Eulerian temporal energy spectrum equation.

By taking the Fourier transform of the transport equation for $Q_{i, i}$, the Eulerian temporal energy spectrum equation can be written as

$$
\frac{D E_{T}}{D t}=\widehat{\mathbb{P}}+\widehat{\mathbb{D}}-\widehat{\mathbb{E}}+\widehat{\mathbb{T}}
$$


The details of the derivation of this equation are given in the appendix.

The terms of the RHS of Eq. (34) are the source and transport terms driving the evolution of the turbulent energy per frequency unit along a streamline. $\widehat{\mathbb{P}}$ is the production by the mean velocity, $\widehat{\mathbb{D}}$ the diffusion term, sum of the turbulent $\widehat{\mathbb{D}}^{T}$, molecular $\widehat{\mathbb{D}}^{\nu}$ and pressure $\widehat{\mathbb{D}}^{P}$ diffusions, and $\widehat{\mathbb{E}}$ the dissipation rate. The term $\widehat{\mathbb{T}}$ originates from the non-linear interactions, and is a spectral flux, i.e., does not contribute to the creation or destruction of turbulent kinetic energy, since

$$
\int_{0}^{\infty} \widehat{\mathbb{T}}(\mathbf{x}, \omega) \mathrm{d} \omega=0
$$

Note that integrating Eq. (34) over all the frequencies, the turbulent kinetic energy equation is obtained

$$
\frac{D k}{D t}=\underbrace{-\overline{u_{i} u_{j}} \frac{\partial U_{i}}{\partial x_{j}}}_{P}+\underbrace{\nu \frac{\partial^{2} k}{\partial x_{j} \partial x_{j}}-\frac{1}{2} \frac{\partial \overline{u_{i} u_{i} u_{j}}}{\partial x_{j}}-\frac{1}{\rho} \frac{\partial \overline{p u_{j}}}{\partial x_{j}}}_{D}-\underbrace{\nu \overline{\frac{\partial u_{i}}{\partial x_{j}} \frac{\partial u_{i}}{\partial x_{j}}}}_{\varepsilon}
$$

Since the subfilter kinetic energy is given by Eq. (33), its transport equation can be obtained by taking the derivative of Eq. (33) and making use of Eq. (34), which leads to

$$
\frac{D k_{m}}{D t}=P_{m}+D_{m}-\varepsilon_{m}-T_{G}
$$

where

$$
\begin{aligned}
& D_{m}=\int_{0}^{\infty}\left(1-\widehat{G}_{\Delta_{T}} \widehat{G}_{\Delta_{T}}^{*}\right) \widehat{\mathbb{D}} \mathrm{d} \omega ; \quad P_{m}=\int_{0}^{\infty}\left(1-\widehat{G}_{\Delta_{T}} \widehat{G}_{\Delta_{T}}^{*}\right)(\widehat{\mathbb{P}}+\widehat{\mathbb{T}}) \mathrm{d} \omega ; \\
& \varepsilon_{m}=\int_{0}^{\infty}\left(1-\widehat{G}_{\Delta_{T}} \widehat{G}_{\Delta_{T}}^{*}\right) \widehat{\mathbb{E}} \mathrm{d} \omega ; \quad T_{G}=\int_{0}^{\infty} E_{T} \frac{D}{D t}\left(\widehat{G}_{\Delta_{T}} \widehat{G}_{\Delta_{T}}^{*}\right) \mathrm{d} \omega .
\end{aligned}
$$

The terms $\varepsilon_{m}$ and $D_{m}$ are respectively the SFS dissipation and diffusion terms. The term $P_{m}$ is the sum of a production term by the mean velocity and a spectral flux. These terms are the subfilter parts of the terms appearing in Eq. (36). Moreover, in the RANS limit, $\Delta_{T} \rightarrow \infty$, Eq. (36) is recovered term by term, since $\lim _{\Delta_{T} \rightarrow \infty} \widehat{G}_{\Delta_{T}}(\omega)=0$. The last term in the right-hand side of Eq. (37), $T_{G}$, is a transfer term arising from the variations of filter width. This term is second order in a standard LES, and, therefore, is usually neglected (Ghosal and Moin, 1995). 


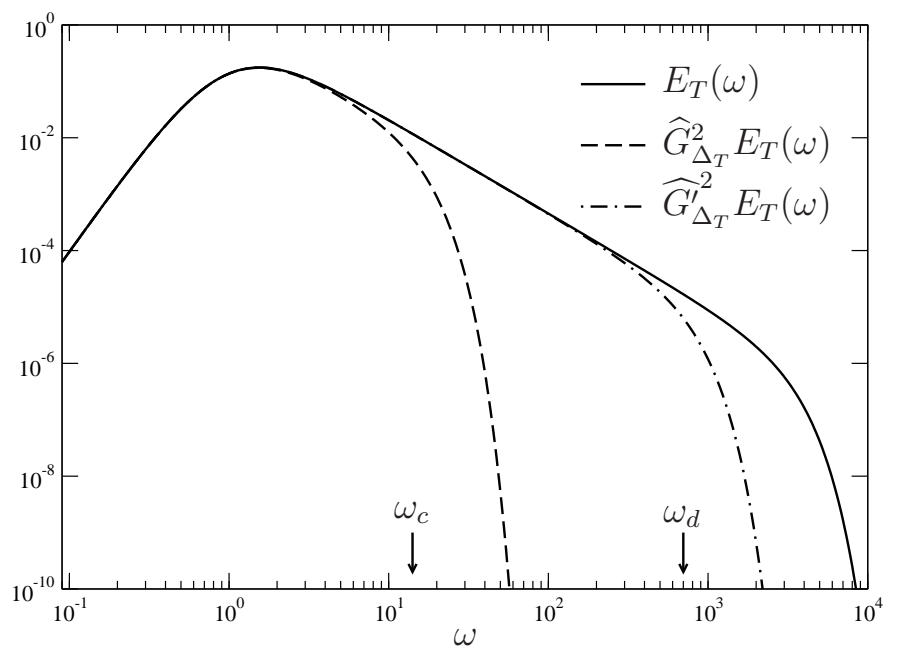

Figure 1: Schematic view of the application of the two filters to the Eulerian temporal spectrum (arbitrary units).

\subsection{Dissipation rate equation}

In seamless hybrid RANS/LES methods, the amount of resolved energy is to be controlled by making the equations of the model dependent on the filter width. Similar to the PITM approach (Schiestel and Dejoan, 2005; Chaouat and Schiestel, 2005) (spatial filtering), this can be achieved by using a transport equation for the dissipation rate that is a modification of the usual RANS equation. In order to know how to modify this equation to make it dependent on the characteristic frequency of the filter $\omega_{c}=\pi / \Delta_{T}$, a second filter $G_{\Delta_{T}}^{\prime}$ is introduced, at the frequency $\omega_{d}$, such that the turbulent spectrum is schematically divided into three parts, the resolved range $\left[0 ; \omega_{c}\right]$, the subfilter energetic range $\left[\omega_{c} ; \omega_{d}\right]$ and the subfilter dissipative range $\left[\omega_{d} ; \infty\right]$, as illustrated in Fig. 1. With this energy partition, the model is a particular case, reduced to only three spectral zones, of the multi-scale models proposed by Schiestel (1983a,b, 1987). Similar to what was proposed by Schiestel (1987) and Schiestel and Dejoan (2005) for the spatial version of the PITM, the following definition is used for $\omega_{d}$

$$
\omega_{d}=\omega_{c}+\chi_{m} \frac{\varepsilon_{m}}{k_{m}}
$$

where $\chi_{m}$ is an arbitrary constant, chosen such a way that the energy in the range $\left[\omega_{d} ; \infty\right]$ is negligible compared to the energy in the range $\left[\omega_{c} ; \omega_{d}\right]$. Using Eq. (37), the material derivative of (40) leads to a transport equation 
for the subfilter dissipation rate

$$
\frac{D \varepsilon_{m}}{D t}=\frac{\varepsilon_{m}}{k_{m}} P_{m}-\frac{\varepsilon_{m}^{2}}{k_{m}}\left[1-\left(\frac{k_{m}}{\varepsilon_{m}}\right) \frac{\dot{\omega}_{d}-\dot{\omega}_{c}}{\omega_{d}-\omega_{c}}+\frac{T_{G}}{\varepsilon_{m}}\right]+\frac{\varepsilon_{m}}{k_{m}} D_{m} .
$$

where $\dot{\phi}$ denotes the material derivative of $\phi$. In order to write Eq. (41) in a form similar to the usual RANS equation, the functions $C_{\varepsilon_{1}}^{\prime}$ and $C_{\varepsilon_{2}}^{\prime}$ are introduced

$$
\frac{D \varepsilon_{m}}{D t}=C_{\varepsilon_{1}}^{\prime} \frac{\varepsilon_{m}}{k_{m}} P_{m}-C_{\varepsilon_{2}}^{\prime} \frac{\varepsilon_{m}^{2}}{k_{m}}+\frac{\varepsilon_{m}}{k_{m}} D_{m}
$$

where

$$
C_{\varepsilon_{2}}^{\prime}=1+\left(C_{\varepsilon_{1}}^{\prime}-1\right) \frac{P_{m}}{\varepsilon_{m}}-\left(\frac{k_{m}}{\varepsilon_{m}}\right) \frac{\dot{\omega}_{d}-\dot{\omega}_{c}}{\omega_{d}-\omega_{c}}+\frac{T_{G}}{\varepsilon_{m}} .
$$

$C_{\varepsilon_{1}}^{\prime}$ can be chosen arbitrarily as a function of the characteristic frequency $\omega_{c}$, but it must satisfy the RANS limiting behavior $\lim _{\omega_{c} \rightarrow 0} C_{\varepsilon_{1}}^{\prime}=C_{\varepsilon_{1}} .\left(C_{\varepsilon_{1}}\right.$ and $C_{\varepsilon_{2}}$ denote hereafter the coefficients entering the dissipation equation used in RANS mode.) Moreover, in order to recover the standard RANS dissipation equation in the RANS limit, the function $C_{\varepsilon_{2}}^{\prime}$ given by Eq. (43) must satisfy

$$
\lim _{\omega_{c}, \dot{\omega}_{c} \rightarrow 0} C_{\varepsilon_{2}}^{\prime}=1+\left(C_{\varepsilon_{1}}-1\right) \frac{P}{\varepsilon}-\frac{k}{\varepsilon} \frac{\dot{\omega}_{d}}{\omega_{d}}=C_{\varepsilon_{2}}
$$

This relation provides a constraint on $\dot{\omega}_{d}$ that can be introduced into Eq. (43) to give

$$
\begin{aligned}
& C_{\varepsilon_{2}}^{\prime}=\frac{r}{r_{\varepsilon}} \frac{\omega_{d}}{\omega_{d}-\omega_{c}}\left(C_{\varepsilon_{2}}-1-\frac{P}{\varepsilon}\left(C_{\varepsilon_{1}}-1\right)\right) \\
& +1+\left(C_{\varepsilon_{1}}^{\prime}-1\right) \frac{P_{m}}{\varepsilon_{m}}+\left(\frac{k_{m} / \varepsilon_{m}}{\omega_{d}-\omega_{c}} \dot{\omega}_{c}+\frac{T_{G}}{\varepsilon_{m}}\right),
\end{aligned}
$$

where $r=k_{m} / k$, and $k=k_{r}+k_{m}$ is the total kinetic energy, i.e., the sum of the resolved part $k_{r}=\frac{1}{2} \overline{u_{i}^{\prime} u_{i}^{\prime}}$ and SFS part $k_{m}=\frac{1}{2} \overline{\tau_{i i S F S}}$. Similarly, $r_{\varepsilon}=\varepsilon_{m} / \varepsilon$, and $\varepsilon=\varepsilon_{r}+\varepsilon_{m}$ is the total dissipation rate, i.e., the sum of the resolved part $\varepsilon_{r}$ and SFS part $\varepsilon_{m}$. At this stage, it can be noticed that Eq. (45) is difficult to use from a practical point of view, because the total (resolved+SFS) variables $P$ and $\varepsilon$ are not known at the beginning of the computation: a relation between these total variables and their resolved part is needed in order to rewrite Eq. (45) in terms of modeled quantities only. Such a relation can be obtained from the material derivative of $r=k_{m} / k$, which gives exactly, using of Eq. (36) and Eq. (37)

$$
\frac{P}{\varepsilon}=1+\frac{r_{\varepsilon}}{r}\left(\frac{P_{m}}{\varepsilon_{m}}-1\right)+\frac{r_{\varepsilon}}{\varepsilon_{m}}\left(\frac{D_{m}}{r}-D\right)-\frac{k_{m}}{\varepsilon_{m}} \frac{r_{\varepsilon}}{r^{2}} \dot{r}-\frac{r_{\varepsilon}}{r} \frac{T_{G}}{\varepsilon_{m}} .
$$


In the following, it will be assumed that the filter width varies sufficiently slowly for the terms in $\dot{\omega}_{c}$ and $T_{G}$ to be negligible in Eq. (45), as well as the terms in $\dot{r}$ and $T_{G}$ in Eq. (46). This hypothesis, e.g. valid for a channel flow, is used here to simplify the analysis, but the influence of the variations of the filter width can be reintroduced if necessary. By inserting Eq. (46) into Eq. (45), the SFS dissipation rate equation can be written as

$$
\frac{D \varepsilon_{m}}{D t}=C_{\varepsilon_{1}}^{\prime} \frac{P_{m} \varepsilon_{m}}{k_{m}}-C_{\varepsilon_{2}}^{\prime} \frac{\varepsilon_{m}^{2}}{k_{m}}+D_{\varepsilon_{m}}
$$

with

$$
\begin{aligned}
C_{\varepsilon_{2}}^{\prime}= & \frac{\omega_{d}}{\omega_{d}-\omega_{c}}\left[\frac{r}{r_{\varepsilon}}\left(C_{\varepsilon_{2}}-C_{\varepsilon_{1}}\right)+\left(C_{\varepsilon_{1}}-1\right) \times\right. \\
& \left.\left(1-\frac{P_{m}}{\varepsilon_{m}}\right)\right]+1+\left(C_{\varepsilon_{1}}^{\prime}-1\right) \frac{P_{m}}{\varepsilon_{m}}
\end{aligned}
$$

and

$$
D_{\varepsilon_{m}}=\frac{\varepsilon_{m}}{k_{m}} D_{m}+\left(C_{\varepsilon_{1}}-1\right) \frac{\varepsilon_{m}}{k_{m}} \frac{\omega_{d}}{\omega_{d}-\omega_{c}}\left(D_{m}-r D\right) .
$$

In practical applications, the aim of such a hybrid model is not to achieve the DNS limit, especially if the Reynolds number is high. Therefore, it is assumed that $\omega_{c} \ll \omega_{d}$ and $r_{\varepsilon} \sim 1$, such that Eq. (48) becomes

$$
C_{\varepsilon_{2}}^{\prime}=C_{\varepsilon_{1}}+r\left(C_{\varepsilon_{2}}-C_{\varepsilon_{1}}\right)+\frac{P_{m}}{\varepsilon_{m}}\left(C_{\varepsilon_{1}}^{\prime}-C_{\varepsilon_{1}}\right)
$$

Inserting Eq. (50) into Eq. (47), it is noticed that the final result does not depend on the function $C_{\varepsilon_{1}}^{\prime}$, since the SFS dissipation rate equation is finally given by

$$
\frac{D \varepsilon_{m}}{D t}=C_{\varepsilon_{1}} \frac{\varepsilon_{m}}{k_{m}} P_{m}-\underbrace{\left[C_{\varepsilon_{1}}+r\left(C_{\varepsilon_{2}}-C_{\varepsilon_{1}}\right)\right]}_{C_{\varepsilon_{2}}^{*}} \frac{\varepsilon_{m}^{2}}{k_{m}}+D_{\varepsilon_{m}}
$$

Eq. (51) is similar to the one found in the spatial PITM approach (Schiestel and Dejoan, 2005; Chaouat and Schiestel, 2005), but includes the additional diffusion term $D_{\varepsilon_{m}}$. The RANS/TLES transition is controlled by the parameter $r$. The RANS limit corresponds to $r=1$, in which case the classical RANS dissipation rate equation is recovered. During the computation, the value of this parameter must be prescribed as a function of the filter width, in order to enforce the desired behavior of the model. This function is proposed in the next section. 


\subsection{Relation between $r$ and the filter width}

In TLES, the choice of the temporal filter width $\Delta_{T}$, or equivalently, the frequency $\omega_{c}=\pi / \Delta_{T}$, is not as obvious as in spatial LES. Transposing the standard practice of spatial LES in the temporal domain, it would seem straightforward to link $\Delta_{T}$ to the time step used in the computation, $\Delta t$, for instance by $\Delta_{T}=2 \Delta t$. However, since $\Delta t$ is necessarily the same everywhere in the domain, this relation does not allow local variations of the filter width, which is in contradiction to the objective of hybrid models. Moreover, it is widely recognized in LES that linking the filter width to the local grid step optimizes the cost of a computation.

Therefore, the possibility of linking the temporal filter width to the local mesh refinement is investigated. For this purpose, $\omega_{c}$ must be related to the wavenumber associated with the mesh size $\kappa_{c}=\pi(\Delta x \Delta y \Delta z)^{-1 / 3}$. The main difficulty then lies in the fact that a dispersion relation $\omega=f(\kappa)$ is required to evaluate the frequency $\omega_{c}$. This relation is not known in general, and combines complex effects. Indeed, the frequency associated to a particular wavenumber in not only determined by the natural lifetime of the structure, but also by their convection velocity (Doppler effect), as mentioned by Pruett (2000).

However, since the Eulerian frequency spectrum $E_{T}(\omega)$ and the wavenumber spectrum $E(\kappa)$ are related by

$$
\mathrm{d} k=E(\kappa) \mathrm{d} \kappa=E_{T}(\omega) \mathrm{d} \omega,
$$

the energy ratio $r=k_{m} / k$, which is the only parameter entering the model, can be evaluated either in the frequency domain or in the wavenumber domain.

In order to obtain a simple analytical expression of $r$, a spectral cutoff filter is considered. In this case, the ratio $r$ is given by

$$
r=\frac{1}{k} \int_{\omega_{c}}^{\infty} E_{T}(\omega) \mathrm{d} \omega .
$$

Using Eq. (52), a change of variable yields

$$
\begin{aligned}
r & =\frac{1}{k} \int_{\kappa_{c}}^{\infty} E_{T}(\omega) \frac{E(\kappa)}{E_{T}(\omega)} \mathrm{d} \kappa \\
& =\frac{1}{k} \int_{\kappa_{c}}^{\infty} E(\kappa) \mathrm{d} \kappa
\end{aligned}
$$

This relation is a noticeable feature of the TPITM formulation: the model only depends on the parameter $r$, which can be evaluated by integration of 
either the temporal spectrum or the spatial spectrum, as shown by Eq. (55). Consequently, the model can be equivalently sensitized to the cutoff frequency of the filter $\omega_{c}$ or to the corresponding wavenumber defined by $\kappa_{c}=$ $f^{-1}\left(\omega_{c}\right)$, without any explicit knowledge of the dispersion relation $f$. This remarks is interesting for two reasons: firstly, the model for the temporally filtered equations can be linked to the local grid step, which is convenient in practice and enables optimizing the use of computer resources; secondly, the ratio $r$ can thus be evaluated using a schematic shape for the wavenumber spectrum, such asa standard Kolmogorov spectrum, which yields

$$
r=\frac{3}{2} C_{\kappa}\left(\kappa_{c} \frac{k^{3 / 2}}{\varepsilon}\right)^{-\frac{2}{3}} .
$$

Such a relation cannot be found in general using Eq. (53), since the Eulerian temporal spectrum is not known. It is worth emphasizing that the use of this relation does not imply that the temporal filtering is replaced by spatial filtering. It is only a convenient way, justified by Eq. (55), of making the equations of the model sensitive to the local grid step, i.e., of adapting the cutoff frequency of the temporal filter to the highest frequency that can be resolved by the grid.

An illustration of this result can be given by considering the particular case of turbulence in the absence of mean flow. In this case, Tennekes (1975) assumed that the scales in the inertial range are swept by the large scales, and proposed the dispersion relation

$$
\omega=\kappa \sqrt{k} .
$$

Using this relation yields

$$
\begin{aligned}
\mathrm{d} k & =C_{\kappa} \varepsilon^{2 / 3} \kappa^{-5 / 3} \mathrm{~d} \kappa \\
& =C_{\kappa} \varepsilon^{2 / 3} k^{1 / 3} \omega^{-5 / 3} \mathrm{~d} \omega,
\end{aligned}
$$

such that

$$
E_{T}(\omega)=C_{\kappa} \varepsilon^{2 / 3} k^{1 / 3} \omega^{-5 / 3} .
$$

Using this Eulerian spectrum in Eq. (53) finally provides the relation between $r$ and the cutoff frequency

$$
r=\frac{3}{2} C_{\kappa}\left(\omega_{c} \frac{k}{\varepsilon}\right)^{-\frac{2}{3}}
$$

which is identical to Eq. (56) since $\omega_{c}=\kappa_{c} \sqrt{k}$. 
Therefore, in the present TPITM formulation, the temporal filter width is not explicitly used. The transport equations for the subfilter stresses are indirectly sensitized to the filter width through the associated dissipation rate equation; the temporal filter implicitly enters the latter via the energy ratio $r$, and the above analysis shows that $r$ can be related to the local grid step.

\section{Example of model based on the TPITM methodology: Elliptic blending model for the subfilter stresses}

The previous section shows that a RANS model can be modified in order to be used as a TLES model by simply making the coefficient $C_{\varepsilon_{2}}$ in the dissipation rate equation a function of the filter width. This procedure can in principle be used within any type of RANS model that uses a dissipation rate equation (the adaptation to the $\omega$ equation is straightforward).

However, in a hybrid RANS/TLES context, in which the cutoff frequency can be located in the large-scale region of the turbulent spectrum, we believe that it is necessary to take into account the complex production and redistribution mechanisms occurring at these scales. Therefore, the use of transport equations for the SFS stresses, rather than an eddy-viscosity model, is desirable, as proposed in the spatial PITM context by Chaouat and Schiestel (2005); Jakirlić et al. (2009).

Since the transport equation for the subfilter-stress tensor (17) is formally identical to the transport equations for the Reynolds-stress tensor, it can be assumed that using a model formally identical to a RANS second-moment closure is adequate, as soon as the length and time scales are modified to account for the variable cutoff frequency.

In this paper, in order to demonstrate the applicability of the TPITM approach, an adaptation of the Elliptic Blending Reynolds-Stress Model (EBRSM) (Manceau and Hanjalić, 2002; Manceau, 2005), usually applied in a RANS context, is used. In this model, six transport equations for the subfilter stresses are thus solved, in addition to the transport equation (51) for the dissipation rate. However, it is worth emphasizing here that Eq. (51) is written for $\varepsilon_{m}$, i.e., the long-time averaged value of the SFS dissipation rate $\varepsilon_{m}=\overline{\varepsilon_{S F S}}=\frac{1}{2} \overline{\varepsilon_{i i S F S}}$, The analysis done in spectral space shows that the variable coefficient $C_{\varepsilon 2}^{*}$ enables the control of the amount of long-time averaged modeled energy $k_{m}=\overline{k_{S F S}}$. In the computations, equations are solved for the time-dependent subgrid stresses rather than for their long-time averages. 
However, it is assumed that the control of the level of modeled energy via $C_{\varepsilon 2}^{*}$ is also valid for the time-dependent quantities: Eq. (51) is thus solved for the time-dependent subfilter dissipation rate

$$
\frac{D \varepsilon_{S F S}}{D t}=C_{\varepsilon_{1}} \frac{\varepsilon_{S F S}}{k_{S F S}} P_{S F S}-C_{\varepsilon_{2}}^{*} \frac{\varepsilon_{S F S}^{2}}{k_{S F S}}+D_{\varepsilon_{S F S}}
$$

Note that the diffusion term is modeled by a standard generalized gradient diffusion hypothesis.

\subsection{Model for the subfilter stresses}

The EB-RSM model is an extension of the SSG model (Speziale et al., 1991) to the near-wall region. The particularity of this model is that it uses a blending of the "quasi-homogeneous" (away from the wall) and the near-wall models of the pressure term $\phi_{i j_{S F S}}$ and the dissipation tensor $\varepsilon_{i j_{S F S}}$ using

$$
\begin{aligned}
\phi_{i j_{S F S}} & =\left(1-\alpha^{3}\right) \phi_{i j}^{w}+\alpha^{3} \phi_{i j}^{h}, \\
\varepsilon_{i j_{S F S}} & =\left(1-\alpha^{3}\right) \frac{\tau_{i j_{S F S}}}{k_{S F S}} \varepsilon_{S F S}+\alpha^{3} \frac{2}{3} \varepsilon_{S F S} \delta_{i j} .
\end{aligned}
$$

$\alpha$ is a blending coefficient which goes from zero at the wall, to unity far from the wall. The nonlocal character of the pressure is reproduced by solving an elliptic equation for the blending function

$$
\alpha-L_{S F S}^{2} \nabla^{2} \alpha=1 \text {. }
$$

The near-wall form of the model $\phi_{i j}^{w}$ can be shown to be consistent with the asymptotic behavior of all the variables at the wall when it is taken as

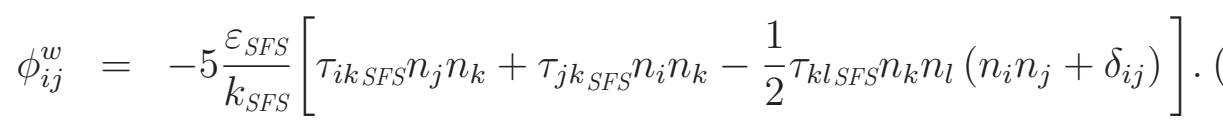

A generalization of the concept of wall-normal vector is used here: $\mathbf{n}=$ $\boldsymbol{\nabla} \alpha /\|\boldsymbol{\nabla} \alpha\|$, which is applicable everywhere in the domain. The SSG model (Speziale et al., 1991) is used for $\phi_{i j}^{h}$.

The association of the elliptic blending model and the TPITM approach leads to some modeling issues that have been previously investigated in the frame of spatial PITM (Fadai-Ghotbi et al., 2007; Jakirlić et al., 2009): in particular, a better control of the parameter $r$ in the near-wall region is obtained when the elliptic blending function $\alpha$ is empirically introduced in the formulation in order to enforce the RANS mode close to the wall

$$
r=\left(1-\alpha^{3}\right)+\alpha^{3} \beta_{0}^{-1}\left(\kappa_{c} \frac{k^{3 / 2}}{\varepsilon}\right)^{-\frac{2}{3}},
$$


with $\beta_{0}=0.20$, and the length scale of the elliptic equation (65) is made a function of the parameter $r$

$$
L_{S F S}=C_{L} \max \left(\frac{k_{S F S}^{3 / 2}}{\varepsilon_{S F S}}, r^{3 / 2} C_{\eta} \frac{\nu^{3 / 4}}{\varepsilon^{1 / 4}}\right) .
$$

In Eqs. (67) and (68), $k$ and $\varepsilon$ are evaluated during the computation, using time-averaging and, in the particular case of the channel flow, averaging in homogeneous directions. $k$ is the sum of the modeled energy $k_{m}$ and the resolved energy $k_{r}$, defined by Eqs. (22) and (23), respectively. Consistent with the hypothesis $r_{\varepsilon}=1$ made in section 4.2 , the contribution of the resolved scales to the dissipation rate is assumed negligible, such that $\varepsilon=\overline{\varepsilon_{S F S}}$. These modeling aspects are directly imported from the spatial PITM adaptation of the EB-RSM and are not repeated herein. The reader is referred to Fadai-Ghotbi et al. (2007); Jakirlić et al. (2009) for details.

\subsection{Validation in channel flow}

Computations are performed using the open-source software Code_Saturne, a parallel, finite volume solver on unstructured grids, developed at EDF (Archambeau et al., 2004), distributed under Gnu GPL license ${ }^{2}$. The numerical method is based on a SIMPLEC algorithm, with a Rhie \& Chow interpolation in the pressure correction step. The convection terms in the transport equations (16) for the filtered momentum are discretized using central differencing. On the contrary, upwind biased differencing is used in the transport equations for the subfilter stresses (17). Time-marching is based on a secondorder, Crank-Nicolson scheme.

The case is a channel flow at $R e_{\tau}=395$, and results are compared with DNS data (Moser et al., 1999). The mesh is made of $32 \times 54 \times 32$ cells, with $\Delta x^{+}=100, \Delta z^{+}=50$ and, at the wall, $\Delta y_{1}^{+}=1.5$, which is very coarse compared to a mesh suitable for LES, which requires with the present secondorder numerical method $\Delta x^{+}=20, \Delta z^{+}=10$ and, at the wall, $\Delta y_{1}^{+}=1$. In order to evaluate the sensitivity of the model to the mesh, a second mesh was also used, consisting of $48 \times 54 \times 48$.

First of all, it is worth pointing out that the hybrid method is indeed able to control the respective contributions of the resolved (filtered) and modeled (subfilter) fields. The relation (67) for the energy ratio $r$ aims at enforcing a RANS solution in the near-wall region, independently of the grid, and at continuously transitioning to TLES toward the outer part of the flow. Fig. 2

\footnotetext{
${ }^{2}$ http://www.code-saturne.org
} 

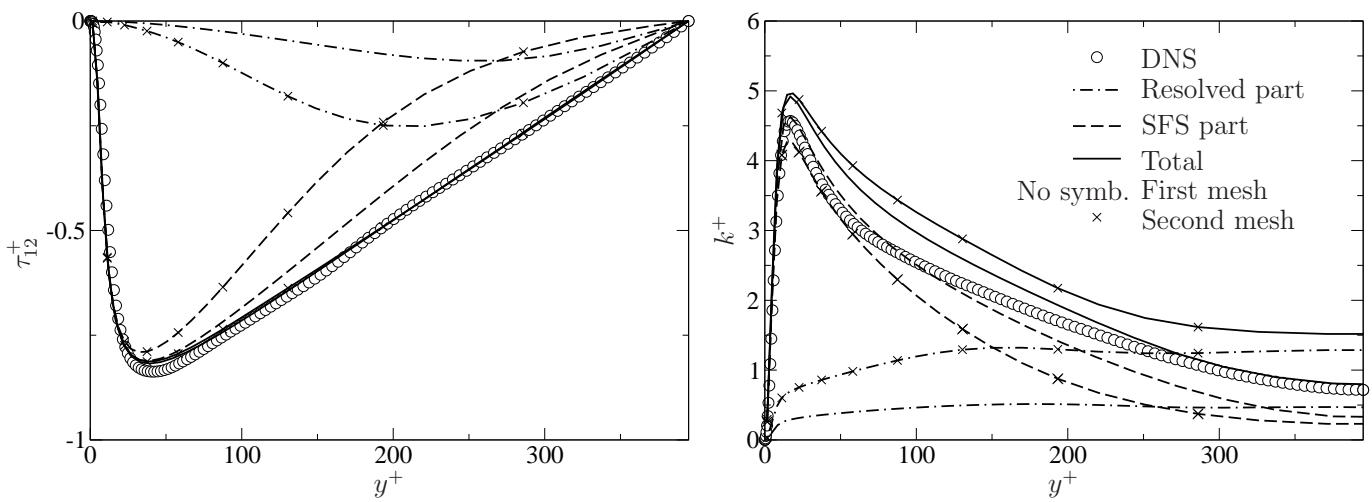

Figure 2: Contributions of the resolved and modeled flow fields to the shear stress (left) and the turbulent energy (right) for the two meshes.

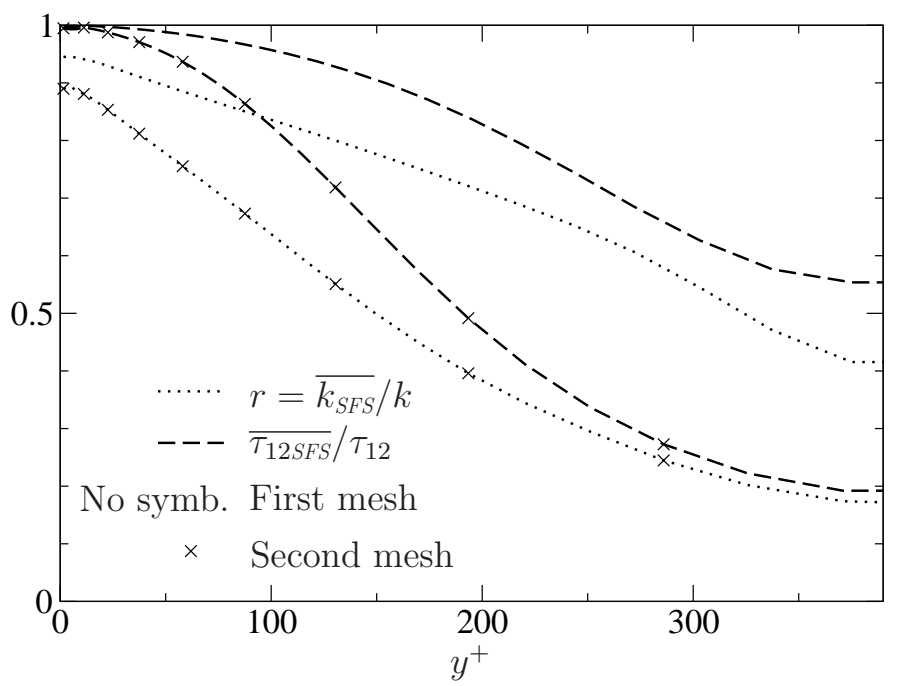

Figure 3: Ratio of modeled energy and shear-stress. 


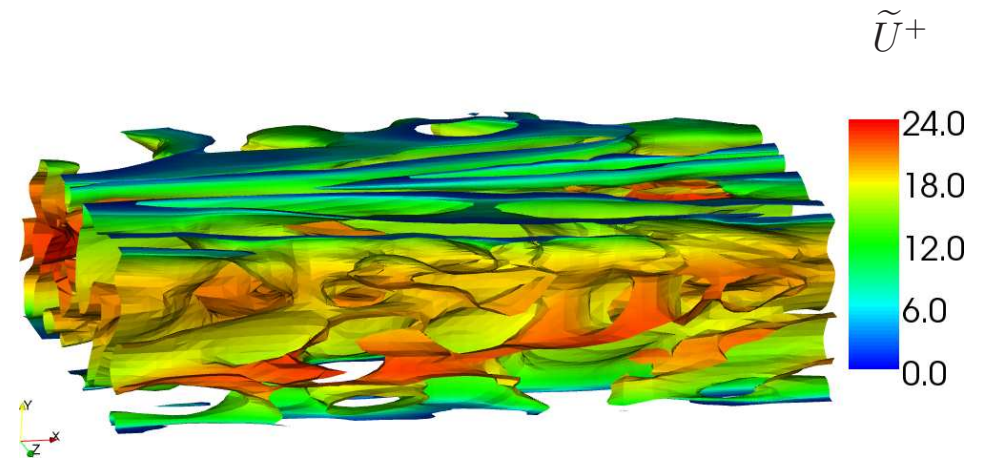

Figure 4: Isocontour $Q=-\frac{1}{2} \frac{\partial \widetilde{U}_{i}}{\partial x_{j}} \frac{\partial \widetilde{U}_{j}}{\partial x_{i}}=0$ colored by the filtered velocity (second mesh).

shows that this aim is fulfilled for both the shear stress and the turbulent kinetic energy. In the near-wall region, the contribution of the resolved stress is zero and the modeled stress represents the integrality of the total shear stress. This partition of the stress progressively transitions toward the center of the channel, where the amount of resolved shear stress reaches about $50 \%$ for the first mesh, and $80 \%$ for the second mesh. The same trend is observed for the turbulent kinetic energy, although the weight of the subfilter part is slightly larger than in the case of the shear stress. Although the partition of energy among the resolved and SFS scales is very dependent on the mesh refinement, the total turbulent energy remains approximately constant, in particular below $y^{+}=250$. A variation in the central region was also observed by Chaouat and Schiestel (2005) with the spatial version of the PITM, using a different model for the subfilter scales. As shown in Fig. 3, the energy ratio $r=k_{m} / k$ (modeled energy/total energy) significantly varies in the domain, and with mesh refinement. At the center of the channel, it reaches about 0.4 for the first mesh and 0.2 for the second mesh, i.e., the contribution of the resolved scales represents $60 \%$ and $80 \%$, respectively. Since in the central region, $\alpha=1$, Eq. (67) shows that $r$ is determined by the local grid step.

A particularly interesting feature can be observed in Fig. 3: the ratio $r=$ $k_{m} / k$ does not reach unity at the wall, contrary to the ratio $\overline{\tau_{12 S F S}} / \tau_{12}$. This behavior shows that the near-wall region is subject to unsteadiness induced by the large scale motions coming from the outer layer. These motions contribute to the fluctuating energy in the near-wall region, but not to the shear stress, which is $100 \%$ modeled at the wall. This description corresponds to the definition of the inactive motions of Townsend. Although it does not provide any detailed information about the dynamics of the flow, the structures present in the resolved field are visualized using the $Q$-criterion in Fig. 4. As could be expected in such a low resolution computation, only very large scale structures are obtained. 


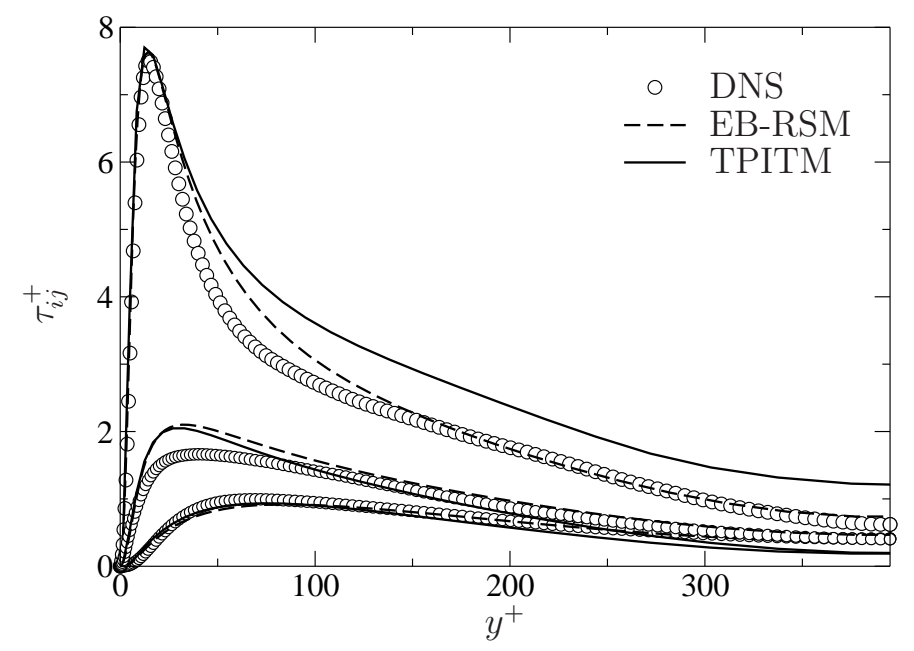

Figure 5: Diagonal Reynolds stresses (first mesh).

Figs. 2 and 5 show a satisfactory reproduction of the turbulent energy and the Reynolds stresses. In the near-wall region, this results is to be credited to the RANS model. In the central region, the contribution of the resolved field is significant, but the level of modeled energy is appropriately reduced, such that the sum of the two contribution provides the correct level of energy. Fig. 5 shows that in the TLES region the streamwise fluctuations are overestimated, to the detriment of the fluctuations in the other directions.Since the RANS model correctly reproduces all the Reynolds stresses in the central region, as can be seen in the figure, this behavior is to be related to the TPITM methodology or, at least, to the use of a modified RANS model as a SFS model.

Finally, Fig. 6 illustrates the main interest of this approach: despite the fact that DNS is not perfectly matched, the mean velocity profile is very acceptable, even using the first mesh which is much coarser than a LEStype mesh. As shown in the figure, a LES performed with the dynamic Smagorinsky model on the same mesh strongly overestimates the flow rate. When the mesh is refined, although the relative weights of the resolved and modeled contributions to the flow field are drastically modified, as shown above, the prediction of the mean velocity profile shows a very moderate sensitivity to the mesh, the variation of the flow rate being less than $0.5 \%$.

\section{Extension to other methodologies}

As mentioned in the Introduction, numerous approaches have been proposed in the recent years to achieve an objective similar to the TPITM presented 


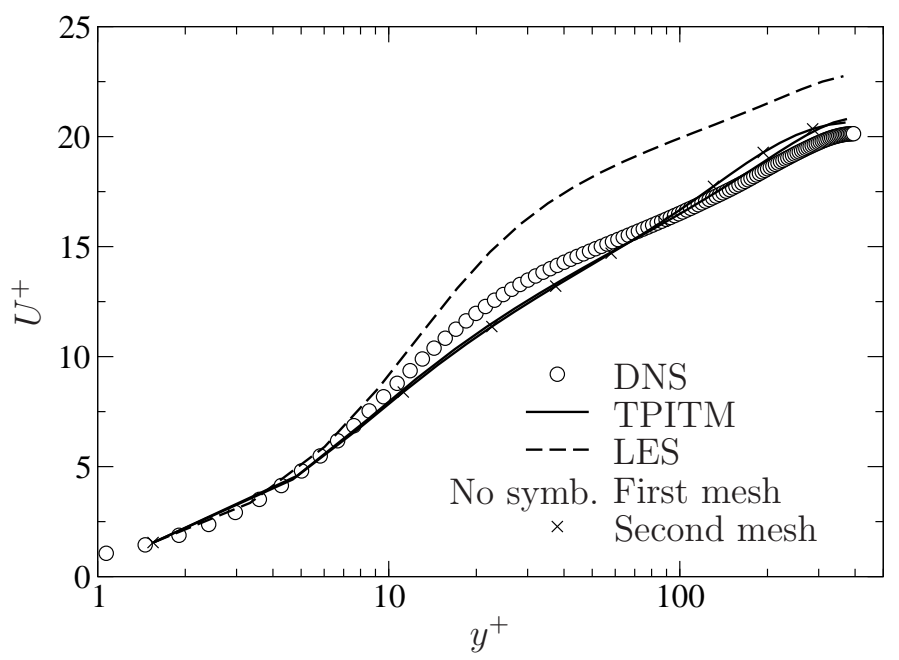

Figure 6: Mean velocity profiles. Comparison with results obtained on the first mesh using the dynamic Smagorinsky model.

in section 4, i.e., to provide a level of description of turbulence intermediate between LES and RANS. They are collectively termed as eddy-resolving methods by Fröhlich and von Terzi (2008). The approaches that ensure a continuous transition from a RANS model to a LES model, depending on the local grid size, are called seamless, global (Sagaut et al., 2006), unified (Fröhlich and von Terzi, 2008) or non-zonal hybrid RANS/LES models. Other methods, which do not rely explicitly on the grid step, are denoted by second-generation URANS models by Fröhlich and von Terzi (2008).

One of the main features shared by these models is an ambiguous definition of the resolved variables, as mentioned in the Introduction. Indeed, in the regions where the model works in LES mode, the spatially-filtered momentum equations are solved. In the RANS region, the Reynolds average is used, which is a statistical average, and the two approaches do not continuously match, except in the case of homogeneous flows.

On the contrary, it was shown in section 2 that the introduction of temporal filtering provides a consistent framework for inhomogeneous, stationary flows. Thus, it suggests that seamless and second-generation URANS methods should be regarded as TLES/RANS hybrid methods.

Indeed, all the models share a common objective: controlling the level of stresses in the momentum equations to ensure a transition from a RANS to a (T)LES behavior where necessary. The TPITM model presented in section 4.2 is based on transport equations for the subfilter stresses $\tau_{i j_{S F S}}$, but an eddy-viscosity TPITM model can be easily derived following the same methodology (similar to the eddy-viscosity PITM model proposed by Schi- 
estel and Dejoan, 2005). In this case, the subfilter viscosity is

$$
\nu_{t}=C_{\mu} \frac{k_{m}^{2}}{\varepsilon}=r^{2} \nu_{t R A N S},
$$

where $\nu_{\text {tRANS }}$ is the eddy-viscosity of a RANS model. In case of spectral equilibrium, for a cutoff frequency in the inertial range, it can be deduced from Eq. (56) that the subfilter length scale is directly linked to the cutoff wavenumber by

$$
\frac{k_{m}^{3 / 2}}{\varepsilon}=\left(\frac{3 C_{\kappa}}{2}\right)^{3 / 2} \kappa_{c}^{-1} \simeq \Delta_{S},
$$

where $\Delta_{S}=\pi / \kappa_{c}$ (the TPITM does not assume equilibrium at the cutoff frequency, such that, in general, the length scale characterizing the largest unresolved structures is determined by the transport equations, and Eq. 70 does not hold). Therefore, in this case, the TPITM is equivalent to a spatial LES with the filter width $\Delta_{S}$.

It is easy to show that other methodologies, using various modifications of the equations, basically achieve the same reduction of the level of stresses in the momentum equation, via a direct damping of the eddy-viscosity or, equivalently, a reduction of the length scale. For instance, the most widespread hybrid method, DES (Spalart et al., 1997), in its original formulation, directly reduces the level of eddy-viscosity in detached regions, by increasing its destruction term. In SAS (Menter et al., 2003), the same effect is achieved by a decrease of the production term in the eddy-viscosity transport equation. Other methods, such as OES (Bourguet et al., 2007, 2008), XLES (Kok et al., 2004) or the method of Fan et al. (2004), directly modify the Boussinesq relation, either by redefining the coefficient $C_{\mu}$ or by blending the RANS and LES formulae. In LNS (Batten et al., 2004) and FSM (Fasel et al., 2002), the stresses in the momentum equations are directly premultiplied by a damping factor, as originally proposed by Speziale (1998), which is equivalent to modifying the eddy-viscosity. In the case of PANS (Girimaji, 2006), which controls the ratio modeled energy/total energy through the $C_{\varepsilon_{2}}$ coefficient, the similarity with PITM is obvious, despite a completely different argumentation and significant differences in the practical details.

Many of these methods are built in such a way that, for sufficiently fine grids, the length scale entering the model coincides with the grid step, in order to be equivalent to LES. However, as shown in section 4.3, a relation can be found between the wavenumber and the frequency characterizing the energy partition, which reflects the fact that a spatial filtering implicitly induces a temporal filtering, and vice versa. Therefore, a hybrid formulation based on a filter width related to the grid step can be associated with a temporallyfiltered approach. 
More precisely, the relation between the temporal filter and the spatial filter can be expressed in a case where the turbulent spectra can be defined both in frequency and wavenumber domains, as, e.g., forced homogeneous turbulence. Indeed, the explicit introduction of the dispersion relation $\omega=f(\kappa)$ into Eq. (52) yields

$$
E(\kappa)=E_{T} \circ f(\kappa) \frac{\mathrm{d} f}{\mathrm{~d} \kappa}(\kappa)
$$

Now, for any temporal filter $G_{\Delta_{T}}$, the resolved energy reads

$$
\begin{aligned}
k_{r} & =\int_{0}^{\infty} \widehat{G}_{\Delta_{T}}(\omega) \widehat{G}_{\Delta_{T}}^{*}(\omega) E_{T}(\omega) \mathrm{d} \omega \\
& =\int_{0}^{\infty} \widehat{G}_{\Delta_{T}} \circ f(\kappa) \widehat{G}_{\Delta_{T}}^{*} \circ f(\kappa) E_{T} \circ f(\kappa) \frac{\mathrm{d} f}{\mathrm{~d} \kappa}(\kappa) \mathrm{d} \kappa
\end{aligned}
$$

Moreover, the corresponding spatial filter must satisfy

$$
k_{r}=\int_{0}^{\infty} \widehat{G}_{\Delta_{S}}(\kappa) \widehat{G}_{\Delta_{S}}^{*}(\kappa) E(\kappa) \mathrm{d} \kappa
$$

such that, using Eq. (71),

$$
\widehat{G}_{\Delta_{S}}=\widehat{G}_{\Delta_{T}} \circ f .
$$

Finally, the case of URANS in stationary flows deserves some attention. Indeed, in such flows, the Reynolds average is equivalent to a long-time average, such that the solution of the RANS equations is expected to be independent of time. However, in practice, e.g. in the case of the wake of a bluff body, the solutions found are often time-dependent, and the method is then denoted by URANS. It is worth emphasizing that, although the RANS equations are recovered by making the temporal filter width go to infinity, the time scale entering the RANS models is the integral time scale. Therefore, the equations of the model are formally identical to the equations of a subfilter stress model with a filter width equal to the integral time scale. Consequently, URANS can be regarded as a particular case of hybrid RANS/TLES. Depending on the practical flow configuration, in particular the type of instability modes at the origin of the appearance of unsteadiness, the solution of this model can be steady or unsteady (i.e., RANS or URANS, respectively). The possibility of obtaining a steady solution with a filter of finite width is not surprising: it is the method used by Akervik et al. (2006) to obtain unstable, steady solutions of the Navier-Stokes equations in globally unstable flows. 


\section{Conclusion}

Temporal filtering provides a consistent formalism for a class of seamless hybrid RANS-LES methodologies, applicable to most of the industrial configurations, which are inhomogeneous and stationary. In such flows, the generalized temporal filter introduced in section 2 consistently bridges the two approaches, RANS, based on Reynolds averaging, and TLES, based on temporal filtering, in a Galilean invariant manner. In practice, similar to what is usually done for approaches developed in homogeneous turbulence, the present approach can also be applied to non-stationary flows, such as flows with variable boundary conditions, although the theoretical link between RANS and TLES is then lost.

The formal similarity between the RANS and TLES equations provides the basis for the use of formally similar models for the subfilter stresses, with a continuous variation of the coefficients of the model to transition between the two methodologies.

In order to seamlessly transition from RANS to TLES, the model must be able to control the amount of resolved energy. Such a model, the TPITM, the temporal version of the PITM, was proposed. It was shown through an analysis in the frequency domain that any RANS model using a transport equation for the dissipation rate can be sensitized to the cutoff frequency introduced by the filter. One of the interests of this approach is that the cornerstone is the energy ratio (ratio between the subfilter and total turbulent energies), which can be expressed either as a function of the cutoff frequency of the filter or as a function of the cutoff wavenumber of the spatial filter implicitly related to the temporal filter. Consequently, the temporal filter width can be implicitly fixed by the grid step, which enables local refinement and optimizes the use of computational resources.

Although the TPITM method can be used with eddy-viscosity models, in order to account for the complex production/redistribution mechanisms occurring when the cutoff frequency lies in the energetic scales, transport equations are solved for the subfilter stresses. The model provides very encouraging predictions of the mean velocity profile and turbulent anisotropy in a channel flow.

Finally, based on the fact that a temporally filtered approach does not necessarily need to use explicitly the temporal filter width, but can rather be based on a corresponding spatial filter width, it can be concluded that most of the existing seamless hybrid methodologies, such as the widely used DES, or the so-called second-generation URANS models (Fröhlich and von Terzi, 2008), such as SAS, and even the basic URANS, can be regarded as temporally filtered approaches. This point of view enables the conciliation of a 
consistent formalism and methods routinely used in applied research.

\section{Appendix: Derivation of the Eulerian tempo- ral spectrum transport equation}

In a stationary flow, the Eulerian temporal energy spectrum $E_{T}$ is the trace of the Fourier transform of the two-time correlation tensor

$$
E_{T}(\mathbf{x}, \omega)=\widehat{Q}_{i, i}(\mathbf{x}, \omega)
$$

with

$$
Q_{i, j}(\mathbf{x}, \tau)=\overline{u_{i}(\mathbf{x}, t) u_{j}(\mathbf{x}, t+\tau)}=\overline{u_{i A} u_{j B}},
$$

where indices $A$ and $B$ denote the values taken at times $t_{A}=t$ and $t_{B}=t+\tau$, respectively. The temporal Fourier transform $\widehat{\phi}$ of any variable $\phi$, and its inverse, are defined as

$$
\begin{aligned}
\widehat{\phi}(\mathbf{x}, \omega) & =\frac{1}{2 \pi} \int_{-\infty}^{+\infty} e^{-i \omega \tau} \phi(\mathbf{x}, \tau) \mathrm{d} \tau \\
\phi(\mathbf{x}, \tau) & =\int_{-\infty}^{+\infty} e^{i \omega \tau} \widehat{\phi}(\mathbf{x}, \omega) \mathrm{d} \omega
\end{aligned}
$$

The turbulent kinetic energy $k=\frac{1}{2} \overline{u_{i} u_{i}}$ is given by

$$
k(\mathbf{x})=\frac{1}{2} Q_{i, i}(\mathbf{x}, 0)=\int_{0}^{\infty} E_{T}(\mathbf{x}, \omega) \mathrm{d} \omega .
$$

Note that for stationary flows, $Q_{i, i}(\tau)$ is an even function, such that $E_{T}(\omega)$ is a real and even function.

The equation of the fluctuating velocity $u_{i}$ leads to

$$
\begin{aligned}
U_{k} \frac{\partial}{\partial x_{k}}\left(u_{i A} u_{j B}\right)= & -u_{j B} \frac{\partial u_{i A}}{\partial t_{A}}-u_{i A} \frac{\partial u_{j B}}{\partial t_{B}}-u_{k A} u_{j B} \frac{\partial U_{i}}{\partial x_{k}}-u_{i A} u_{k B} \frac{\partial U_{j}}{\partial x_{k}} \\
& -u_{i A} \frac{\partial}{\partial x_{k}}\left(u_{j B} u_{k B}-\overline{u_{j B} u_{k B}}\right)-u_{j B} \frac{\partial}{\partial x_{k}}\left(u_{i A} u_{k A}-\overline{u_{i A} u_{k A}}\right) \\
& -\frac{u_{j B}}{\rho} \frac{\partial p_{A}}{\partial x_{i}}-\frac{u_{i A}}{\rho} \frac{\partial p_{B}}{\partial x_{j}}+\nu u_{j B} \frac{\partial^{2} u_{i A}}{\partial x_{k} \partial x_{k}}+\nu u_{i A} \frac{\partial^{2} u_{j B}}{\partial x_{k} \partial x_{k}}
\end{aligned}
$$

In stationary flows, the mean velocity does not depend on time, and therefore 
$U_{k A}=U_{k B}=U_{k}$. The following definitions are introduced:

$$
\begin{gathered}
\mathcal{S}_{i k, j}(\mathbf{x}, \tau)=\overline{u_{i A} u_{k A} u_{j B}} \quad ; \quad \mathcal{E}_{i, j}(\mathbf{x}, \tau)=2 \nu \frac{\overline{\partial u_{i A}} \frac{\partial u_{j B}}{\partial x_{k}}}{\partial x_{k}} \\
K_{(p), j}(\mathbf{x}, \tau)=\overline{p_{A} u_{j B}} \quad ; \quad K_{i,(p)}(\mathbf{x}, \tau) \\
\mathcal{T}_{i, j}(\mathbf{x}, \tau)=\overline{\left(u_{k A}-u_{k B}\right) u_{i A} \frac{\partial u_{j B}}{\partial x_{k}}}
\end{gathered}
$$

in which subscripts before and after the comma refer to the times $t_{A}=t$ and $t_{B}=t+\tau$, respectively. The subscript $(p)$ refers to the pressure, and is not a tensorial subscript. Using the incompressibility constraint, simple algebra yields

$$
\overline{-u_{i A} \frac{\partial}{\partial x_{k}} u_{j B} u_{k B}}-\overline{u_{j B} \frac{\partial}{\partial x_{k}} u_{i A} u_{k A}}=-\frac{\partial \mathcal{S}_{i k, j}}{\partial x_{k}}+\mathcal{T}_{i, j}
$$

and

$$
\overline{\nu u_{j B} \frac{\partial^{2} u_{i A}}{\partial x_{k} \partial x_{k}}}+\nu \overline{\nu u_{i A} \frac{\partial^{2} u_{j B}}{\partial x_{k} \partial x_{k}}}=\nu \frac{\partial^{2} Q_{i, j}}{\partial x_{k} \partial x_{k}}-\mathcal{E}_{i, j}
$$

Introducing the change of variables $t=t_{A}$ and $\tau=t_{B}-t_{A}$

$$
\begin{aligned}
\frac{\partial}{\partial t_{A}} & =\frac{\partial}{\partial t} \frac{\partial t}{\partial t_{A}}+\frac{\partial}{\partial \tau} \frac{\partial \tau}{\partial t_{A}}=\frac{\partial}{\partial t}-\frac{\partial}{\partial \tau} \\
\frac{\partial}{\partial t_{B}} & =\frac{\partial}{\partial t} \frac{\partial t}{\partial t_{B}}+\frac{\partial}{\partial \tau} \frac{\partial \tau}{\partial t_{B}}=\frac{\partial}{\partial \tau},
\end{aligned}
$$

it can be shown that

$$
\overline{u_{j B} \frac{\partial u_{i A}}{\partial t_{A}}}+\overline{u_{i A} \frac{\partial u_{j B}}{\partial t_{B}}}=\left(\frac{\partial}{\partial t_{A}}+\frac{\partial}{\partial t_{B}}\right) Q_{i, j}=\frac{\partial Q_{i, j}}{\partial t}=0
$$

Taking the Reynolds average of Eq. (81), and using relations (83), (84) and (87), the transport equation for the two-time correlation tensor is obtained

$$
\begin{aligned}
\frac{D Q_{i, j}}{D t}= & -\frac{\partial U_{i}}{\partial x_{k}} Q_{k, j}-\frac{\partial U_{j}}{\partial x_{k}} Q_{i, k}-\frac{\partial \mathcal{S}_{i k, j}}{\partial x_{k}}+\mathcal{T}_{i, j}+\nu \frac{\partial^{2} Q_{i, j}}{\partial x_{k} \partial x_{k}}-\mathcal{E}_{i, j} \\
& -\frac{1}{\rho}\left(\frac{\partial K_{i,(p)}}{\partial x_{j}}+\frac{\partial K_{(p), j}}{\partial x_{i}}\right)+\frac{1}{\rho}\left(\overline{p_{B} \frac{\partial u_{i A}}{\partial x_{j}}}+\overline{p_{A} \frac{\partial u_{j B}}{\partial x_{i}}}\right) .
\end{aligned}
$$

where $D / D t=U_{k} \partial_{k}$.

The trace of Eq. (88) reads

$$
\frac{D Q_{i, i}}{D t}=\mathbb{P}+\underbrace{\mathbb{D}^{\nu}+\mathbb{D}^{T}+\mathbb{D}^{P}}_{\mathbb{D}}-\mathbb{E}+\mathbb{T},
$$


where

$$
\begin{gathered}
\mathbb{P}=-\left(Q_{i, j}+Q_{j, i}\right) \frac{\partial U_{i}}{\partial x_{j}} \quad ; \quad \mathbb{D}^{\nu}=\nu \frac{\partial^{2} Q_{i, i}}{\partial x_{j} \partial x_{j}} \\
\mathbb{D}^{T}=-\frac{\partial \mathcal{S}_{i k, i}}{\partial x_{k}} \quad ; \quad \mathbb{D}^{P}=-\frac{1}{\rho} \frac{\partial}{\partial x_{j}}\left(K_{j,(p)}+K_{(p), j}\right) \\
\mathbb{E}=\mathcal{E}_{k, k} \quad ; \quad \mathbb{T}=\mathcal{T}_{k, k} .
\end{gathered}
$$

For $\tau=0$ (i.e., $t_{A}=t_{B}$ ), Eq. (89) leads to the equation for the turbulent kinetic energy

$$
\frac{D k}{D t}=\underbrace{-\overline{u_{i} u_{j}} \frac{\partial U_{i}}{\partial x_{j}}}_{P}+\underbrace{\nu \frac{\partial^{2} k}{\partial x_{j} \partial x_{j}}-\frac{1}{2} \frac{\partial \overline{u_{i} u_{i} u_{j}}}{\partial x_{j}}-\frac{1}{\rho} \frac{\partial \overline{p u_{j}}}{\partial x_{j}}}_{D}-\underbrace{\nu \overline{\frac{\partial u_{i}}{\partial x_{j}} \frac{\partial u_{i}}{\partial x_{j}}}}_{\varepsilon}
$$

The Fourier transform of Eq. (89) gives

$$
\frac{D E_{T}}{D t}=\widehat{\mathbb{P}}+\widehat{\mathbb{D}}-\widehat{\mathbb{E}}+\widehat{\mathbb{T}}
$$

Integrating Eq. (92) over all the frequencies, the turbulent kinetic energy equation (91) is recovered, since

$$
\begin{aligned}
P(\mathbf{x}) & =\frac{1}{2} \mathbb{P}(\mathbf{x}, 0)=\int_{0}^{\infty} \widehat{\mathbb{P}}(\mathbf{x}, \omega) \mathrm{d} \omega \\
D(\mathbf{x}) & =\frac{1}{2} \mathbb{D}(\mathbf{x}, 0)=\int_{0}^{\infty} \widehat{\mathbb{D}}(\mathbf{x}, \omega) \mathrm{d} \omega, \\
\varepsilon(\mathbf{x}) & =\frac{1}{2} \mathbb{E}(\mathbf{x}, 0)=\int_{0}^{\infty} \widehat{\mathbb{E}}(\mathbf{x}, \omega) \mathrm{d} \omega, \\
0 & =\frac{1}{2} \mathbb{T}(\mathbf{x}, 0)=\int_{0}^{\infty} \widehat{\mathbb{T}}(\mathbf{x}, \omega) \mathrm{d} \omega .
\end{aligned}
$$




\section{References}

Åkervik, E., Brandt, L., Henningson, D., Hoepffner, J., Marxen, O., Schlatter, P., 2006. Steady solutions of the Navier-Stokes equations by selective frequency damping. Phys. Fluids 18 (6).

Antoni, J., 2009. Cyclostationarity by examples. Mech. Syst. Signal Pr. 23 (4), 987-1036.

Archambeau, F., Méchitoua, N., Sakiz, M., 2004. Code Saturne: A finite volume code for the computation of turbulent incompressible flows - Industrial applications. Int. J. on Finite Volumes, Electronical edition: http://averoes.math.univ-paris13.fr/html ISSN 1634 (0655).

Bardina, J., Ferziger, J. H., Reynolds, W. C., 1980. Improved subgrid-scale models for large-eddy simulation. Paper No. 2003-0767, AIAA.

Batten, P., Goldberg, U., Chakravarthy, S., 2004. Interfacing statistical turbulence closures with large-eddy simulation. AIAA J. 42 (3), 485-492.

Bourguet, R., Braza, M., Harran, G., El Akoury, R., 2008. Anisotropic Organised Eddy Simulation for the prediction of non-equilibrium turbulent flows around bodies. J. Fluid Struct. 24 (8), 1240-1251.

Bourguet, R., Braza, M., Perrin, R., Harran, G., 2007. Anisotropic eddyviscosity concept for strongly detached unsteady flows. AIAA J. 45 (5), 1145-1149.

Chaouat, B., Schiestel, R., 2005. A new partially integrated transport model for subgrid-scale stresses and dissipation rate for turbulent developping flows. Phys. Fluids 17, 1-19.

Chaouat, B., Schiestel, R., 2009. Progress in subgrid-scale transport modelling for continuous hybrid non-zonal RANS/LES simulations. Int. J. Heat Fluid Flow 30 (4), 602-616.

Fadai-Ghotbi, A., Manceau, R., Borée, J., 2007. A seamless hybrid RANSLES model based on transport equations for the subgrid stresses and elliptic blending. In: Proc. 5th Symp. Turb. Shear Flow Phenomena, Munich, Germany. pp. 741-746.

Fan, C.-C., Xiao, X., Edwards, J., Hassan, H., Baurle, R., 2004. Hybrid large-eddy/Reynolds-averaged Navier-Stokes simulations of shockseparated flows. Journal of Spacecraft and Rockets 41 (6), 897-906.

Fasel, H., Seidel, J., Wernz, S., 2002. A methodology for simulations of complex turbulent flows. J. Fluids Eng.-T. ASME 124 (4), 933-942.

Fröhlich, J., von Terzi, D., 2008. Hybrid LES/RANS methods for the simulation of turbulent flows. Prog. in Aerosp. Sci. 44 (5), 349-377. 
Germano, M., 1992. Turbulence: the filtering approach. J. Fluid Mech. 238, 325-336.

Ghosal, S., Moin, P., 1995. The basic equations of the large eddy simulation of turbulent flows in complex geometries. J. Comput. Phys. 118, 24-37.

Girimaji, S. S., 2006. Partially-averaged Navier-Stokes model for turbulence: A Reynolds-averaged Navier-Stokes to direct numerical simulation bridging method. J. Appl. Mech. 73 (3), 413-421.

Jakirlić, S., Manceau, R., Sarić, S., Fadai-Ghotbi, A., Kniesner, B., Carpy, S., Kadavelil, G., Friess, C., Tropea, C., Borée, J., 2009. Numerical Simulation of Turbulent Flows and Noise Generation. Notes on Numerical Fluid Mechanics and Multidisciplinary Design. Springer, Ch. LES, Zonal and Seamless Hybrid LES/RANS: Rationale and Application to Free and Wall-Bounded Flows involving Separation and Swirl, pp. 253-282.

Kampé De Fériet, J., Betchov, R., 1951. Theoretical and experimental averages of turbulent functions. Proc. K. Ned. Akad. Wet. 53, 389-398.

Kok, J., Dol, H., Oskam, H., van der Ven, H., 2004. Extra-large eddy simulation of massively separated flows. Paper No. 04-0264, AIAA.

Ma, X., Karamanos, G.-S., Karniadakis, G., 2000. Dynamics and lowdimensionality of a turbulent near wake. J. Fluid Mech. 410, 29-65.

Manceau, R., 2005. An improved version of the Elliptic Blending Model. Application to non-rotating and rotating channel flows. In: Proc. 4th Int. Symp. Turb. Shear Flow Phenomena, Williamsburg, Virginia, USA. pp. 259-264.

Manceau, R., Hanjalić, K., 2002. Elliptic Blending Model: a new near-wall Reynolds-stress turbulence closure. Phys. Fluids 14 (2), 744-754.

Menter, F., Kuntz, M., Bender, R., 2003. A scale-adaptive simulation model for turbulent flow predictions. Paper No. 2003-0767, AIAA.

Moser, R. D., Kim, J., Mansour, N. N., 1999. Direct numerical simulation of turbulent channel flow up to $R e_{\tau}=590$. Phys. Fluids 11 (4), 943-945.

Perrin, R., Braza, M., Cid, E., Cazin, S., Barthet, A., Sevrain, A., Mockett, C., Thiele, F., 2007. Obtaining phase averaged turbulence properties in the near wake of a circular cylinder at high reynolds number using POD. Exp. Fluids 43 (2-3), 341-355.

Pruett, C. D., 2000. Eulerian time-domain filtering for spatial large-eddy simulation. AIAA J. 38 (9), 1634-1642.

Pruett, C. D., Gatski, T. B., Grosch, C. E., Thacker, W. D., 2003. The temporally filtered Navier-Stokes equations: properties of the residual stress. Phys. Fluids 15 (8), 2127-2140. 
Sagaut, P., Deck, S., Terracol, M., 2006. Multiscale and multiresolution approaches in turbulence. Imperial College Press.

Schiestel, R., 1983a. Sur le concept d'échelles multiples en modélisation des écoulements turbulents (partie I). J. Mech. Theor. Appl. 2 (3), 417-449.

Schiestel, R., 1983b. Sur le concept d'échelles multiples en modélisation des écoulements turbulents (partie II). J. Mech. Theor. Appl. 2 (4), 601-628.

Schiestel, R., 1987. Multiple-time-scale modelling of turbulent flows in one point closures. Phys. Fluids 30, 722-731.

Schiestel, R., Dejoan, A., 2005. Towards a new partially integrated transport model for coarse grid and unsteady turbulent flow simulations. Theor. Comput. Fluid Dyn. 18, 443-468.

Spalart, P., Jou, W.-H., Strelets, M., Allmaras, S., 1997. Comments on the feasibility of LES for wings, and on a hybrid RANS/LES approach. In: Liu, C., Liu, Z. (Eds.), First AFOSR International Conference on DNS/LES, 48 August, Ruston, LA, Advances in DNS/LES, Greyden Press, Columbus, OH, USA. pp. 137-147.

Speziale, C. G., 1987. On the decomposition of turbulent flow fields for the analysis of coherent structures. Acta Mech. 70 (1-4), 243-250.

Speziale, C. G., 1998. Turbulence modeling for time-dependent RANS and VLES: a review. AIAA J. 36 (2), 173.

Speziale, C. G., Sarkar, S., Gatski, T. B., 1991. Modeling the pressurestrain correlation of turbulence: an invariant dynamical system approach. J. Fluid Mech. 227, 245-272.

Stolz, S., Adams, N., Kleiser, L., 2001. An approximate deconvolution model for large-eddy simulation with application to incompressible wall-bounded flows. Phys. Fluids 13 (4), 997-1015.

Tejada-Martinez, A. E., Grosch, C. E., Gatski, T. B., 2007. Temporal large eddy simulation of unstratified and stably stratified turbulent channel flows. Int. J. Heat Fluid Flow 28, 1244-1261.

Tennekes, H., 1975. Eulerian and Lagrangian time microscales in isotropic turbulence. J. Fluid Mech. 67, 561-567. 